\title{
Morphological patterns of indirect choroidal rupture on spectral domain optical coherence tomography
}

This article was published in the following Dove Press journal:

Clinical Ophthalmology

22 July 2013

Number of times this article has been viewed

\author{
Unnikrishnan Nair \\ Manoj Soman' \\ Sunil Ganekal ${ }^{2}$ \\ Vaishnavi Batmanabane' \\ KGR Nair' \\ 'Chaithanya Eye Hospital and \\ Research Institute, Trivandrum, Kerala, \\ ${ }^{2}$ Nayana Super Specialty Eye Hospital \\ and Research Center, Davangere, \\ Karnataka, India
}

Purpose: To evaluate the morphological types of indirect choroidal rupture (ICR) using spectral domain optical coherence tomography (SD-OCT).

Methods: This was a prospective interventional study of 18 eyes of 18 patients who presented with a history of blunt ocular trauma resulting in choroidal rupture. All patients underwent detailed ophthalmic evaluation and SD-OCT examination.

Results: Mean age of the patients was $32 \pm 9.6$ years. Morphologically, two types of choroidal rupture were seen on SD-OCT. The first type seen (Type 1 ICR) was a forward protrusion of the retinal pigment epithelium-choriocapillaris (RPE-CC) layer with an acutely angled pyramid or dome shape. This was associated with either a small loss of continuity of the retinal pigment epithelium layer or elevated RPE-CC projection accompanied by a significant quantity of subretinal hemorrhage. The second type observed (Type 2 ICR) was a larger area of disruption of the RPE-CC layer, photoreceptor inner segment/outer segment junction, and external limiting membrane, with a posteriorly directed concave contour depression at that area and downward sliding of tissues into the defect. At presentation, ten eyes were observed to have Type 1 ICR and eight to have Type 2 ICR. Of the 18 eyes, one with Type 1 ICR and two with Type 2 ICR developed choroidal neovascularization (16.6\%).

Conclusion: Two distinct tomographic patterns of choroidal ruptures were identified on SD-OCT, which may allow ruptures to be classified into two morphological types. There are morphometric and clinical differences between the two types, which may help to prognosticate visual outcome and anticipate complications following choroidal ruptures.

Keywords: SD-OCT, ICR, blunt ocular trauma, choroidal neovascularization

\section{Introduction}

Blunt ocular trauma can cause a spectrum of dramatic ocular pathologies ranging from mild to grave. Approximately 5\%-10\% of patients with such injury develop a choroidal rupture. ${ }^{1}$ Indirect choroidal ruptures (ICRs), which result from concussional force $^{2}$ transmitted through the wall of the globe, are discontinuities in the choroid, Bruch's membrane, and the retinal pigment epithelium (RPE). Blunt trauma to the globe momentarily deforms it and, while the tensile strength of the sclera and the elasticity of the retina aids resistance to injury, Bruch's membrane is breached because it is fragile and cannot withstand such a force. Concomitantly, the small capillaries of the choriocapillaris are damaged, leading to subretinal or sub-RPE hemorrhage, while the deep choroidal vessels are usually spared. Ruptures occurring secondary to punch injuries are usually situated in the peripapillary region, while projectile injury commonly produces ruptures in the posterior pole. ${ }^{3}$ Those involving the macula are
Chaithanya Eye Hospital and

Research Institute, Trivandrum,

Kerala, India, 695004

Tel $+9 \mid$ 47I 2447183

Fax+9| 47| 2443985

Email dr.unninair@gmail.com 
predictably associated with a poor visual prognosis and are more predisposed to form choroidal neovascular membranes (CNVMs) ${ }^{4}$ This is an important cause of visual loss, although there are also other reasons for poor visual outcome. ${ }^{5}$ While acute visual loss in eyes with ICR may be due to ruptures passing through the fovea or a rupture associated subretinal hemorrhage extending to lie under the fovea, delayed vision loss occurs because of scar formation or later formation of CNVMs. It is of note that multiple choroidal ruptures are not necessarily associated with a bad visual outcome. ${ }^{6}$

Choroidal neovascularization (CNV) is part of the normal healing process after choroidal trauma. Studies show that there can be a spontaneous involution of the $\mathrm{CNV}$ within weeks following injury; ${ }^{2}$ but, unfortunately, in some eyes, the $\mathrm{CNV}$ is aggressive or gets reactivated, subsequently leading to visual loss in about $5 \%$ of ruptures. ${ }^{7}$ Risk factors for development of CNV include an ICR involving the macula, longer length of ruptures, ruptures closer to the center of the fovea, and those occurring in those of older age. ${ }^{4,8}$

While the clinical and angiographic appearance of ICRs have been well described, ${ }^{9}$ the pathoanatomy of choroidal rupture using spectral domain optical coherence tomography (SD-OCT) has yet to be fully explored. SD-OCT has revolutionized the approach to and understanding of several retinal conditions. Visual reconstruction of retinal pathology is possible due to the high-resolution and volumetric scan modalities available in the latest-generation optical coherence tomography machines. The aim of the study reported here was to analyze the different morphological appearances of ICRs on SD-OCT imaging and to classify them based on the presenting morphology. We also studied the association of clinical features, causative injury, dimensions, locations, and complications, such as subretinal hemorrhage and CNV formation, with each type of ICR in these eyes.

\section{Materials and methods}

This study was a prospective interventional study of 18 eyes of 18 patients who had sustained blunt ocular trauma believed to be the cause of a choroidal rupture, between March 2009 and March 2011. The patients attended the retina clinic in our tertiary care hospital. The study was approved by the Chaitanya Eye Hospital and Research Institute review board and ethics committee. Written informed consent was obtained from all patients. Patients who presented within a week of trauma were included in the study, so that the ICR morphology could be studied at the acute stage. A thorough clinical history was taken from and ophthalmological examination performed on all patients.
The nature of injury and the approximate size of the object causing the trauma, whether smaller or larger than the size of the anterior orbit, were noted. Visual acuity was assessed using a Snellen chart, with results converted to $\log M A R$ values for analysis. Anterior segment examination was performed using slit-lamp biomicroscopy and Goldmann applanation tonometry was performed on all eyes. Posterior segment evaluation included fundus examination with slit-lamp biomicroscopy using a Volk 78D lens and indirect ophthalmoscopy using a Volk 20D lens (Volk Optical, Inc, Mentor, OH, USA). Patients were excluded if there was significant media haze due to vitreous hemorrhage or corneal involvement, precluding morphometric measurements and SD-OCT imaging at presentation or at any time during follow-up.

Any patient presenting later than a week after sustaining injury, requiring surgical management, or for whom less than 6-months' follow-up was achieved was also not included in the analysis. A choroidal rupture was classified as "macular," "foveal," or "peripheral" according to the criteria described by Ament et al. ${ }^{4}$ Other coexistent ocular injuries were also noted. SD-OCT was performed for the involved eyes using a Cirrus ${ }^{\circledR}$ HD-OCT (Carl Zeiss Meditec, Jena, Germany). Cube and raster scans were done through the entire length of the ICR, with image signal strength being in excess of $5 / 10$. Each cube scan consisted of 512 vertical sections and 128 horizontal sections at $5 \mu \mathrm{m}$ cuts. The morphological appearance of the choroidal rupture, changes in the outer retinal layers and choriocapillaris complex, and presence of subretinal hemorrhage were noted. Care was taken to keep the lateral borders of the choroidal ruptures within the scan area through their entire length. All patients had a fundus photograph taken to document the choroidal rupture (using a

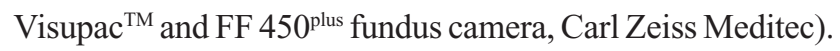
The length of the rupture and the shortest distance between the rupture and the foveal center were measured using the caliper tool of the SD-OCT by a single observer (UN). In case of multiple ruptures, the length of the longest rupture was recorded. In curvilinear ruptures, the free-hand tool was used to measure the linear length. If the rupture passed through the fovea, the proximity from fovea measurement was taken as zero. Fundus fluorescein angiography was done at the point of clinical suspicion of CNVM development.

Statistical analysis was done using SPSS (v 16.0; IBM, Armonk, NY, USA). The unpaired $t$-test was used to compare the means of values between the two groups of ICR, while Fisher's test was used to determine the statistical significance of the size of the projectiles and the number of ruptures in these eyes between the two groups. 


\section{Results}

Of the 18 patients included in our study, 14 were male and four female. The mean age was $32 \pm 9.6$ years (range 10-55 years). Best-corrected visual acuity ranged from counting fingers to Snellen acuity 20/20 (logMAR vision equivalent 0 ). Mean $\log$ MAR vision was 0.750 ( \pm standard deviation [SD] 0.51). The average duration of follow-up for these patients was $17.86 \pm 5.4$ months. Demographic characteristics and anterior segment examination features are described in Table 1.

The choroidal ruptures were clinically apparent as irregular curvilinear breaks in the choroid with associated subretinal hemorrhage (Figure 1A, C, and E). Fundus evaluation revealed choroidal rupture involving the fovea in seven of the 18 eyes. All eyes had ruptures posterior to the equator. Multiple sites of choroidal rupture were seen in five eyes. One patient who developed a peripheral traumatic cataract was included in analysis, as the view and imaging of the retina was not hampered.

\section{SD-OCT features}

Two patterns of choroidal rupture were identified on SD-OCT. The first pattern (Type 1 ICR) was a forward protrusion of the retinal pigment epithelium-choriocapillaris (RPE-CC) layer with an acute pyramid or dome shape. This was associated with either a small loss of continuity of the RPE layer or a break in the wall of the elevated RPE-CC herniation, along with a variable quantity of subretinal hemorrhage (Figure 1B, D, and F). Reflectivity under the dome seemed to be variable.

The second type (Type 2 ICR) comprised a posteriorly concave area of disruption of the RPE-CC (Figure 2A-F). This was associated with loss of photoreceptor inner segment/ outer segment and external limiting membrane reflectivity

Table I Patient demographics and anterior segment features

\begin{tabular}{lll}
\hline Patient demographics & Number & Percentage \\
\hline Males & 14 & 77.77 \\
Females & 4 & 22.22 \\
Mean age & $32 \pm 9.6$ & \\
& years & \\
Number of eyes injured by a large projectile & 6 & 33.33 \\
Number of eyes injured by a small projectile & 12 & 66.66 \\
Clinical anterior segment features & & \\
Bulbar conjunctival congestion & 18 & 100 \\
Subconjunctival hemorrhage & 3 & 16.6 \\
Corneal abrasion & 3 & 16.6 \\
Anterior uveitis & 12 & 66.6 \\
Hyphema & 2 & 11.11 \\
Traumatic cataract & 1 & 5.55 \\
\hline
\end{tabular}

(Figure 3A-D). There was apparent sliding down of the overlying retinal layers into the defect.

In our study, ten eyes had Type 1 ICR and eight eyes had Type 2 ICR. The eyes were categorized into each type based on their SD-OCT feature at presentation. There was no scenario in which the two types of ICR coexisted, either in the same rupture or in the same eye. When the length of the rupture was perused, each ICR was found to have maintained its integral features over time and there was no change over the follow-up period from one type to the other. The morphometric and SD-OCT features of each type are summarized in Table 2.

Based on their morphology, the two types of ICR were analyzed for associations with mode of injury, number and location of ruptures, and subretinal hemorrhage involvement of the fovea. Table 3 illustrates the frequency of various characteristics in these two categories.

There was no statistically significant difference in mean final visual acuity or mean length of follow-up between the two groups of ICRs. The ten eyes with Type 1 ICR had a mean final vision of 20/31 (Log MAR 0.1903) after a mean follow-up period of 15.1 months. The eight eyes with Type 2 ICR achieved a mean final vision of 20/47 (Log MAR 0.3710) over a mean duration of follow-up of 16.2 months.

Three eyes (16.66\%) developed CNVMs secondary to choroidal rupture; two of these patients had Type 2 ICR on SD-OCT. Of the three eyes with CNVMs, two patients had subfoveal and one had one juxtafoveal location of CNV. All the three patients had Type $2 \mathrm{CNV}$ and their eyes were treated with intravitreal Bevacizumab. The length of the choroidal ruptures in all the three patients was more than $4 \mathrm{~mm}$ and within 1-2 disc diameter from center of the fovea. Associated posterior segment features included optic disc pallor along with choroidal rupture (five eyes), vitreous hemorrhage (four eyes), vitreous base avulsion (one eye), and a peripheral retinal tear (one eye).

\section{Discussion}

Our study revealed that the morphological appearances of ICR on SD-OCT were classifiable into two main types, hitherto unreported to the best of our knowledge.

We noted a predominance of male patients, which was consistent with previous work. ${ }^{3,9}$ In these studies, the main mode of injury was a projectile object not bigger than the diameter of the orbit. A variety of culpable scenarios, ranging from injuries in road traffic accidents to falling fruit, ${ }^{10}$ have been noted, similar to those reported by our patients. Of the six patients who reported injury as a result of a larger 


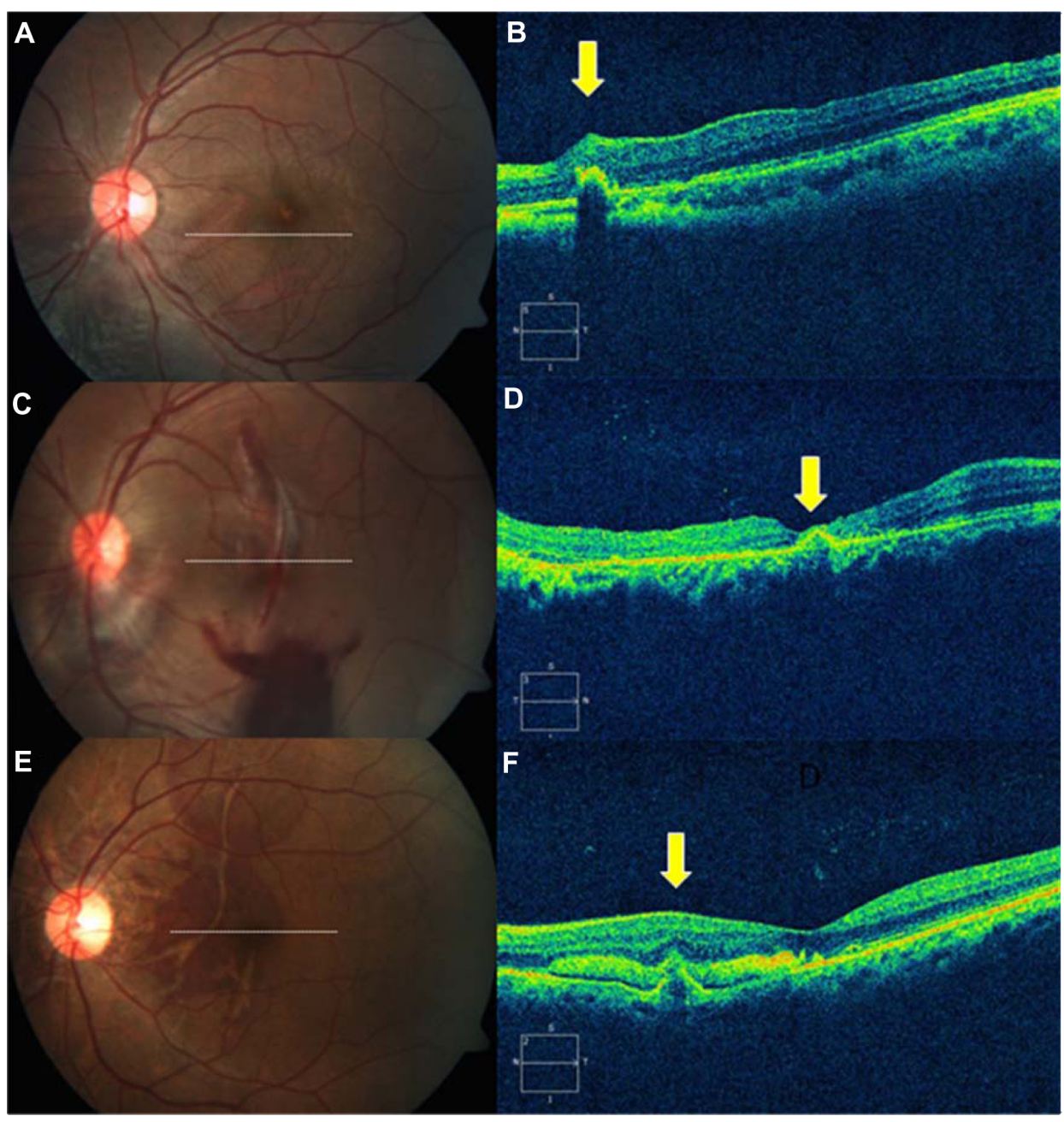

Figure I Type I indirect choroidal rupture (ICR). (A) Extrafoveal ICR nasal to the fovea. (B) Domed-shape elevation with loss of continuity at the base of dome. (C) Multiple ICRs at the macula involving fovea. (D) Subfoveal rupture with apparently intact retinal pigment epithelium-choriocapillaris layer. (E) Multiple ruptures, just sparing the fovea, with subretinal hemorrhage. (F) Extrafoveal pyramid-shape elevation with break in the tip and subfoveal hemorrhage.

projectile, five (83.3\%) had Type 1 ICR, while seven of the twelve $(58.33 \%)$ patients who had been injured by a smaller projectile had Type 2 ICR. Conversely, $87.5 \%$ of the patients who had Type 2 ICRs reported being injured by smaller projectiles. However, the influence of the velocity of the projectile and the force of the trauma were not analyzed, as these parameters were expected to be highly subjective.

Previous studies have described two distinct clinical patterns of ruptures that give rise to CNVMs. ${ }^{3,10-12}$ These clinical patterns correspond to partial thickness ruptures situated temporal to the optic nerve head and curving through the fovea or those situated superiorly or inferiorly around the optic disc, nearly impinging onto the fovea. ${ }^{3}$ This curvilinear configuration is probably a result of maximal stress at these areas. ${ }^{3,13}$ The authors also stress that the location of the rupture is important to note, since the development of CNVMs is likely to be perifoveal ${ }^{3}$ because the RPE is unable to inhibit neovascularization effectively around the fovea. ${ }^{14}$

Our data show that Type 2 ICRs are more likely to be longer, although our findings in this respect did not reach statistical significance when compared with the lengths of the Type 1 ICRs. However, the proximity of Type 2 ruptures to the fovea and the likelihood of being located within the vascular arcades were significantly higher than in the Type 1 ruptures. As is expected, the involvement of the fovea due to subretinal blood was also significantly more common in Type 2 ruptures. However, we could not conclusively prove that foveal ruptures were more common in Type 2 ICRs. This might have been due to the inadequate number of eyes in each group. Yet, we may speculate that Type 2 ruptures are more likely to involve the fovea, due to their being preferentially located within the arcades, and can logically be more often associated with poorer vision and CNVM formation, as discussed 


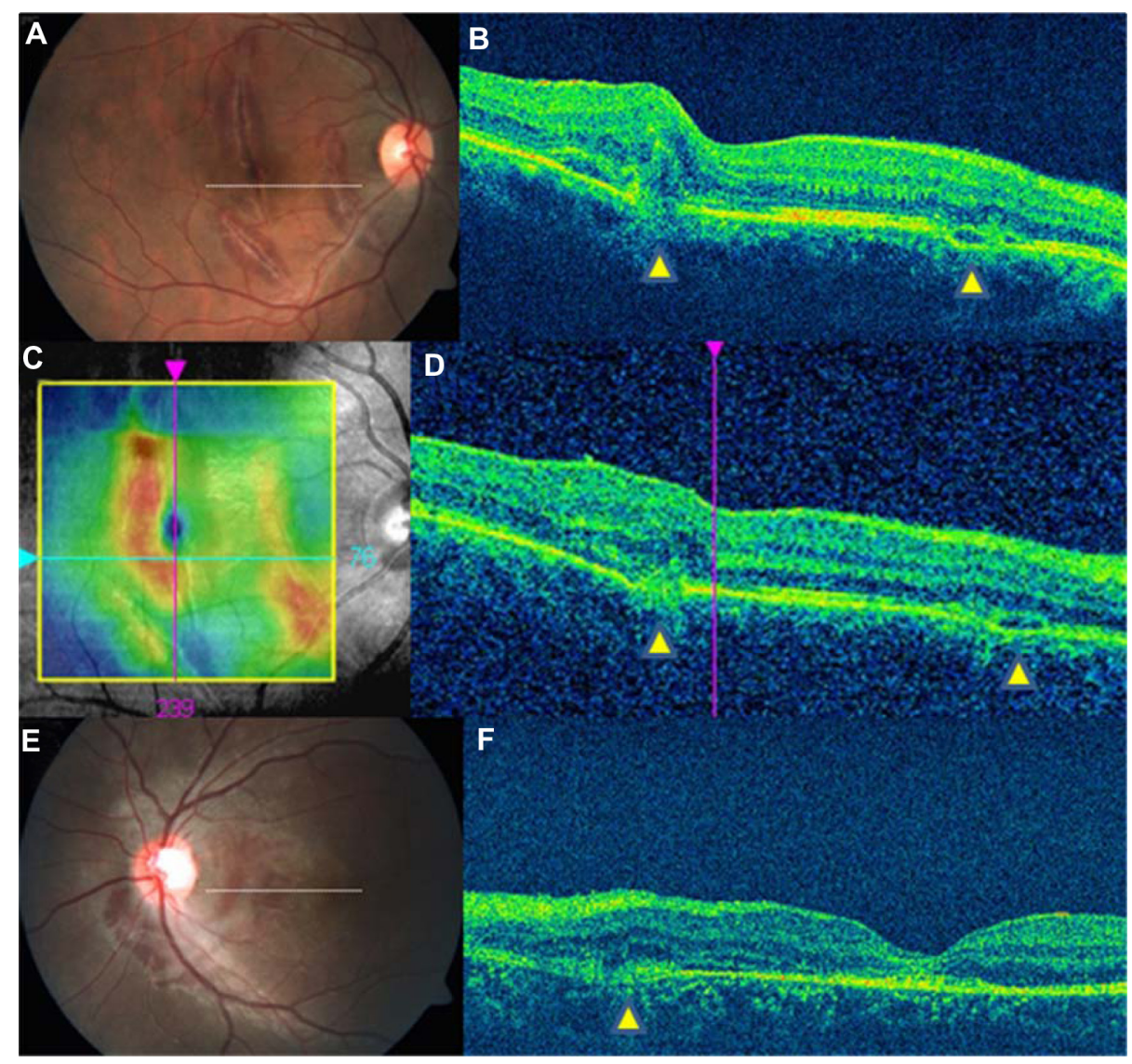

Figure 2 Type 2 indirect choroidal rupture (ICR). (A) Multiple macular ICRs. (B) Loss of continuity of the retinal pigment epithelium (RPE)-choriocapillaris (CC) and inner segment/outer segment layers with subretinal heme. Note the two sites of ruptures (yellow arrowheads). (C) RPE-ILM thickness map, which clearly delineates the rupture site. (D) Horizontal line scan further inferiorly in the same eye, showing no change in the nature of the ICRs (yellow arrowheads). (E) Peripapillary macular ICR. (F) Horizontal line scan at the level indicated in E. The posterior sagging of the retinal layers with loss of the RPE-CC is seen nasal to the fovea (yellow arrowhead). Abbreviation: ILM, internal limiting membrane.

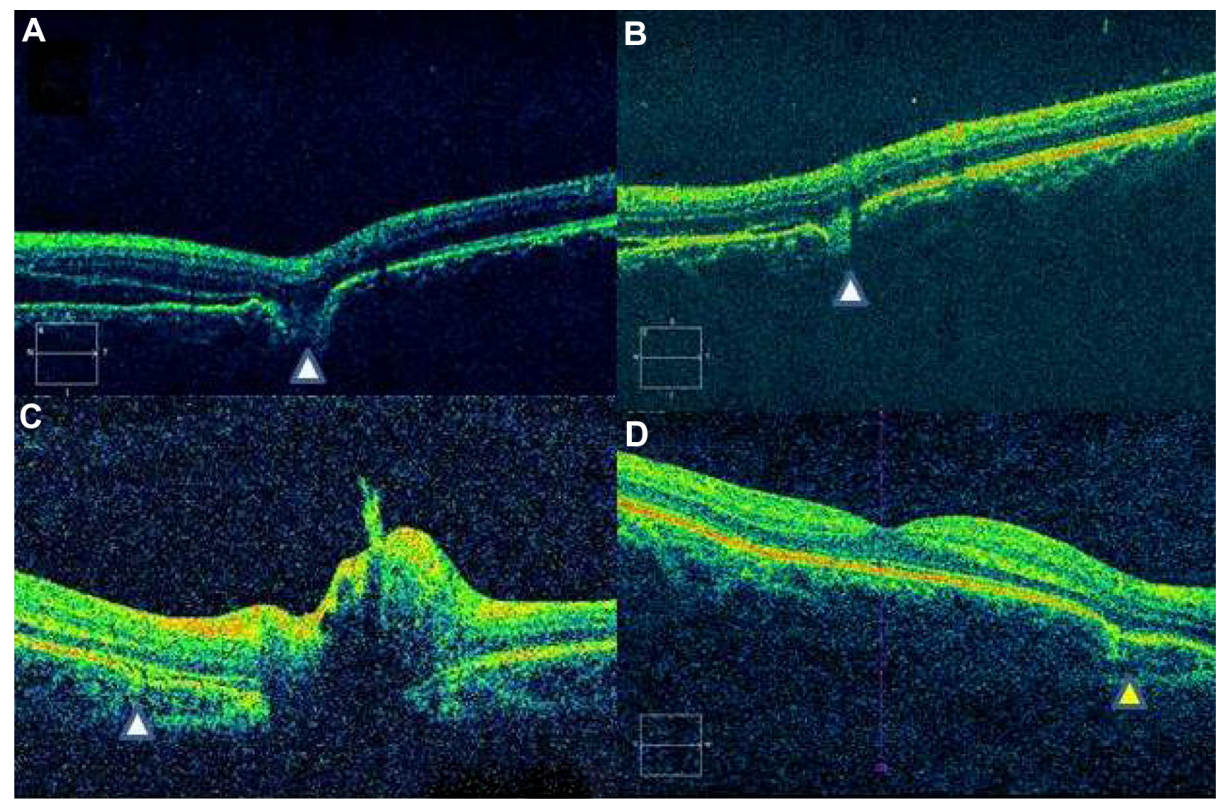

Figure 3 Spectral domain optical coherence tomography features of Type 2 indirect choroidal rupture (ICR). (A) Loss of continuity of the retinal pigment epitheliumchoriocapillaris and inner segment/outer segment layers and ELM with downward sliding of inner retinal layers. (B) Focal loss of continuity with no change in the contour of the overlying retinal layers. (C) Peripapillary choroidal rupture with subretinal bleed. (D) Two months after healing, there is persistence of depression and change in the retinal contour (yellow arrowhead).

Abbreviation: ELM, external limiting membrane. 
Table 2 Morphometric characteristics of indirect choroidal rupture (ICR)

\begin{tabular}{|c|c|c|c|}
\hline Characteristic & $\begin{array}{l}\text { Type I } \\
(n=10)\end{array}$ & $\begin{array}{l}\text { Type } 2 \\
(n=8)\end{array}$ & $P$ \\
\hline $\begin{array}{l}\text { Mean length of } \\
\mathrm{ICR} \text {, } \mathrm{mm}\end{array}$ & $6.86 \pm 2.5$ & $9.18 \pm 5.1$ & $\begin{array}{l}0.22(95 \% \mathrm{Cl} \text {, } \\
-6.2 \text { to } \mathrm{I} .5\end{array}$ \\
\hline $\begin{array}{l}\text { Mean proximity to } \\
\text { fovea, }{ }^{*} \mathrm{~mm}\end{array}$ & $5.11 \pm 3.27$ & $2.59 \pm 1.67$ & $\begin{array}{l}0.06(95 \% \mathrm{Cl}, \\
-0.18 \text { to } 5.2)\end{array}$ \\
\hline
\end{tabular}

previously. ${ }^{3,14}$ In our study, we noted that 12 out of 18 patients had ICRs within the arcades. Of these, the three patients who developed CNVMs had ruptures situated within $1 \mathrm{~mm}$ of the foveal center, in accordance with the previously stated study. ${ }^{4}$ Among these three patients, one had a Type 1 ICR; thus, as we felt that any statistical analysis would be affected by such a small sample, it was not performed. Regardless, it is likely that Type 2 ICRs are more inclined to develop CNV because the amount of tissue disruption that occurs in Type 2 ICR is much more extensive than in Type 1.

In our study, we described SD-OCT features pertinent to the morphological appearance of these ruptures that, as far as we are aware, no previous investigators have described. The peak and trough configurations are likely to be a result of the varying stress-release patterns during blunt ocular trauma. Based on our observation that a Type 1 ICR is more likely to occur as a result of ocular trauma caused by large projectiles, while Type 2 ICRs seem to be more commonly associated with projectiles smaller than the anterior diameter of the orbit, there may be an association between the nature and amount of force to ocular tissues and type of ICR, although in our analysis this did not reach statistical significance. The exact reason for this peculiar configuration of the breaks is largely speculative, but we can hypothesize that some of the velocity of the larger projectiles was deflected by the anterior orbital rim, resulting

Table 3 Clinical characteristics of indirect choroidal ruptures (ICRs)

\begin{tabular}{llll}
\hline & $\begin{array}{l}\text { Type I } \\
(\mathbf{n}=\mathbf{I 0})\end{array}$ & $\begin{array}{l}\text { Type 2 } \\
(\mathbf{n}=\mathbf{8})\end{array}$ & $\mathbf{P}$ \\
\hline Eyes injured by a large projectile, $\mathrm{n}$ & $5(50)$ & $\mathrm{I}(\mathrm{I} 2.5)$ & 0.15 \\
Eyes injured by a small projectile, $\mathrm{n}$ & $5(50)$ & $7(87.5)$ & \\
Single ICR & $7(70)$ & $6(75)$ & 1.0 \\
Multiple ICRs & $3(30)$ & $2(25)$ & \\
ICR located within arcades & $4(40)$ & $8(100)$ & 0.012 \\
ICR located outside arcades & $6(60)$ & 0 & \\
ICR around optic disc & $2(20)$ & $\mathrm{I}(12.5)$ & 1.0 \\
Foveal involvement of subretinal & $5(50)$ & $8(100)$ & 0.012 \\
hemorrhage & & & \\
Foveal involvement of rupture & $4(40)$ & $3(37.5)$ & 1.0 \\
\hline
\end{tabular}

in less of a compressive force and more of a shockwave effect, and therefore less direct trauma to the ocular structures. This translates into a generalized increase in intraorbital pressure with a restricted re-expansion of the globe due to the tamponading effect of the large surface area of a large projectile at the orbital rim. The resultant implosion-type effect leads to a peaked rupture configuration directed internally. In contrast, the large concave depressions seen in Type 2 ruptures may have resulted from the more direct compressive effect and unhindered re-expansion of the globe following compressive trauma with projectiles with smaller surface areas with no capacity to restrict such tissue deformation, thereby causing more extensive tissue damage and splitting of ocular coats.

There is a possibility that because of the greater area of RPE and Bruch's disruption that occurs in Type 2 ICRs that it may be more conducive to the formation of CNV. It has already been established that CNVMs are associated with a poor visual outcome, ${ }^{5}$ with the prognosis further deteriorating if there is foveal involvement.

\section{Conclusion}

Two distinct tomographic patterns of choroidal ruptures were identified on SD-OCT, which may allow ruptures to be classified into two morphological types. This SD-OCT classification may be of practical prognostic value. We would recommend that eyes with a Type 2 ICR need close monitoring with serial SD-OCT for early detection of CNV. However, we found no statistically significant difference in mean final visual acuity between the two groups of ICRs. Our study of 18 eyes allowed us to closely scrutinize the morphology of ICRs and development of complications non-invasively over time. Nevertheless, our small sample size does not permit us to comment on the statistical significance of our findings. A prospective study with a larger number of eyes and longer follow-up may further substantiate our positive findings. Longer follow-up of the patients will determine if the type of ICR, based on our discussion, had a bearing on long-term visual outcome. All patients with an ICR should undergo serial SD-OCT examination for detection of ICR type to help prognosticate vision in eyes that have sustained blunt ocular trauma.

\section{Disclosure}

The authors report no conflicts of interest in this work.

\section{References}

1. Bellows JG. Observations on 300 consecutive cases of ocular war injuries. Am J Ophthalmol. 1947;30(3):309-323.

2. Aguilar JP, Green WR. Choroidal rupture. A histopathological study of 47 cases. Retina. 1984;4(4):269-275. 
3. Wood CM, Richardson J. Indirect choroidal ruptures: aetiological factors, patterns of ocular damage, and final visual outcome. Br J Ophthalmol. 1990;74(4):208-211.

4. Ament CS, Zacks DN, Lane AM, et al. Predictors of visual outcome and choroidal neovascular membrane formation after traumatic choroidal rupture. Arch Ophthalmol. 2006;124(7):957-966.

5. Archer DB, Canavan YM. Contusional eye injuries: retinal and choroidal lesions. Aust J Ophthalmol. 1983;11(4):251-264.

6. Raman SV, Desai UR, Anderson S, Samuel MA. Visual prognosis in patients with traumatic choroidal rupture. Can J Ophthalmol. 2004;39(3): 260-266.

7. Hart CD, Raistrick R. Indirect choroidal tears and late onset serosanguinous maculopathies. Graefes Arch Clin Exp Ophthalmol. 1982;218(4):206-210.

8. Secrétan M, Sickenberg M, Zografos L, Piguet B. Morphometric characteristics of traumatic choroidal ruptures associated with neovascularization. Retina. 1998;18(1):62-66.
9. Hart JC, Natsikos VE, Raistrick ER, Doran RM. Indirect choroidal tears at the posterior pole: a fluorescein angiographic and perimetric study. Br J Ophthalmol. 1980;64(1):59-67.

10. Smith RE, Kelley JS, Harbin TS. Late macular complications of choroidal ruptures. Am J Ophthalmol. 1974;77(5):650-658.

11. Hilton GF. Late serosanguineous detachment of the macula after traumatic choroidal rupture. Am J Ophthalmol. 1975;79(6):997-1000.

12. Pearlstone AD. Delayed loss of central vision following multiple posterior segment trauma. Ann Ophthalmol. 1980;12(4):409-411.

13. Schepens CL. In: Freeman HM, editor. Ocular Trauma. New York, NY: Appleton Century Crofts; 1979:273-284.

14. Bressler NM, Bressler SB, Gragoudas ES. Clinical characteristics of choroidal neovascular membranes. Arch Ophthalmol. 1987;105(2): 209-213.
Clinical Ophthalmology

\section{Publish your work in this journal}

Clinical Ophthalmology is an international, peer-reviewed journal covering all subspecialties within ophthalmology. Key topics include: Optometry; Visual science; Pharmacology and drug therapy in eye diseases; Basic Sciences; Primary and Secondary eye care; Patien Safety and Quality of Care Improvements. This journal is indexed on

Submit your manuscript here: http://www.dovepress.com/clinical-ophthalmology-journal

\section{Dovepress}

PubMed Central and CAS, and is the official journal of The Society of Clinical Ophthalmology (SCO). The manuscript management system is completely online and includes a very quick and fair peer-review system, which is all easy to use. Visit http://www.dovepress.com/ testimonials.php to read real quotes from published authors. 